\title{
THE COMPACT HANKEL OPERATORS FORM AN $M$-IDEAL IN THE SPACE OF HANKEL OPERATORS
}

\author{
DANIEL H. LUECKING
}

\begin{abstract}
The theorem in the title is proved and used to give a new proof that elements of $L^{\infty}$ have best approximations in $H^{\infty}+C$.
\end{abstract}

1. Introduction. Let $L^{\infty}(|z|=1, d \theta)$ denote the usual Lebesgue space of functions on the unit circle. Let $H^{\infty}$ denote the subalgebra consisting of boundary values of bounded analytic functions on $|z|<1$, and $H^{\infty}+C$ the linear span of $H^{\infty}$ and the continuous functions on $|z|=1$. In [3] Axler, et al. prove the following theorem.

TheOREM 1.1. Let $f \in L^{\infty}$. Then there exists $h \in H^{\infty}+C$ such that $\|f-h\|_{\infty}=$ $\operatorname{dist}\left(f, H^{\infty}+C\right)$.

Here $\|\cdot\|_{\infty}$ denotes the essential supremum norm and the distance is measured in this norm.

In this paper we prove the result in the title, which yields 1.1 as a corollary.

2. Preliminaries and main theorem.

Definition. A subspace $J$ of a Banach space $Y$ is called an $L$-ideal if there is projection $E: Y \rightarrow J$ such that

$$
\|y\|=\|E y\|+\|y-E y\|, \quad y \in Y .
$$

Such an $E$ is called an $L$-projection. A subspace $K$ of a Banach space $X$ is called an $M$-ideal if the annihilator $K^{\perp}$ is an $L$-ideal of $X^{*}$.

These concepts may be found in Alfsen and Effros [2]. The property of $M$-ideals we are concerned with is the following.

THeOReM 2.1. If $K$ is an $M$-ideal of $X$ and if $x \in X$, then there exists $m \in K$ such that $\|x-m\|=\operatorname{dist}(x, K)$.

Proof. This is an immediate corollary of Corollary 5.6 of [2]. Although [2] concerns only real Banach spaces and real linear operators one need only consider the underlying real structure to obtain the same result for complex Banach spaces.

We let $H^{2}$ denote the usual Hardy space of functions in $L^{2}(|z|=1, d \theta)$ with vanishing negative Fourier coefficients, and $\left(H^{2}\right)^{\perp}$ the orthogonal complement of $H^{2}$ in $L^{2}$. For $f \in L^{\infty}$ the Hankel operator $H_{f}: H^{2} \rightarrow\left(H^{2}\right)^{\perp}$ is defined by

$$
H_{f} g=P(f g), \quad g \in H^{2},
$$

Received by the editors June 26, 1979.

AMS (MOS) subject classifications (1970). Primary 30A98, 46E15; Secondary 41 A50.

Key words and phrases. $M$-ideal, Hankel operator, best approximation. 
where $P$ is the orthogonal projection onto $\left(H^{2}\right)^{\perp}$. We need only the following properties of Hankel operators.

TheOREM 2.2. The norm of $H_{f}$ is given by $\left\|H_{f}\right\|=\operatorname{dist}\left(f, H^{\infty}\right)$. The essential norm of $H_{f}$ (distance to the compacts) is given by $\left\|H_{f}\right\|_{e}=\operatorname{dist}\left(f, H^{\infty}+C\right)$. In particular, $H_{f}$ is compact if and only if $f \in H^{\infty}+C$.

The first part is due to Nehari [7], the second follows from Theorem 0.1 of [1] (see also [3, p. 608]).

The following is our major result.

THEOREM 2.3. The space of compact Hankel operators forms an M-ideal in the space of all Hankel operators.

Using Theorem 2.2 this may be restated.

THEOREM 2.4. $\left(H^{\infty}+C\right) / H^{\infty}$ is an $M$-ideal in the Banach space $L^{\infty} / H^{\infty}$.

Proof. The map which takes $f+H^{\infty}$ to $H_{f}$ is an isometry of $L^{\infty} / H^{\infty}$ onto the space of Hankel operators that takes $\left(H^{\infty}+C\right) / H^{\infty}$ onto the compact Hankel operators.

We take time out here to show how Theorem 1.1 follows from Theorem 2.1 and Theorem 2.4. These imply that for any $f \in L^{\infty}$ there exists $g \in H^{\infty}+C$ such that $\operatorname{dist}\left(f, H^{\infty}+C\right)=\operatorname{dist}\left(f-g, H^{\infty}\right)$. Since $H^{\infty}$ is weak ${ }^{*}$ closed in $L^{\infty}$, a compactness argument yields $h_{0} \in H^{\infty}$ such that $\operatorname{dist}\left(f-g, H^{\infty}\right)=\left\|f-g-h_{0}\right\|_{\infty}$. This gives us Theorem 1.1 with $h=g+h_{0}$.

3. Proof of the theorem. We assume known a number of standard results in the theory of function algebras (particularly logmodular algebras). Excellent sources are [4], [5], and [6].

To prove the main theorem (in the form Theorem 2.4) we make a number of canonical identifications.

We identify $L^{\infty}$ with $C(M)$ where $M$ is the maximal ideal space (Gelfand space) of $L^{\infty}$. When it is necessary to make a distinction we let $\hat{f} \in C(M)$ denote the function identified with $f \in L^{\infty}$ via the Gelfand transformation.

The dual space $\left(L^{\infty} / H^{\infty}\right) *$ is identified with the space

$$
\left(H^{\infty}\right)^{\perp}=\left\{\mu \in C(M)^{*}: \int f d \mu=0 \text { for all } f \in H^{\infty}\right\}
$$

where $H^{\infty}$ is viewed as a subalgebra of $C(M)$. As such it is logmodular (see [5]).

We identify $\left(\left(H^{\infty}+C\right) / H^{\infty}\right)^{\perp}$ with $\left(H^{\infty}+C\right)^{\perp}=\left\{\mu \in C(M)^{*}: \int f d \mu=0\right.$ for all $\left.f \in H^{\infty}+C\right\}$. We point out that $H^{\infty}+C$ is a closed subalgebra of $L^{\infty}$. (See [8, p. 191].)

Let $m$ denote the lifting of Lebesgue measure from the unit circle to $M$. That is,

$$
\int_{M} \hat{f} d m=\frac{1}{2 \pi} \int_{0}^{2 \pi} f\left(e^{i \theta}\right) d \theta, \text { for } f \in L^{\infty} .
$$

This induces an isomorphism of $L^{1}(|z|=1, d \theta)$ with $L^{1}(M, d m)$ in which (3.1) holds for $f \in L^{1}$. 
To prove Theorem 2.4 we have to produce an $L$-projection of $\left(H^{\infty}\right)^{\perp}$ onto $\left(H^{\infty}+C\right)^{\perp}$. It is constructed as follows. A measure $\mu \in\left(H^{\infty}\right)^{\perp}$ can be written $\mu=\mu_{a}+\mu_{s}$ where $\mu_{a}$ is absolutely continuous with respect to $m$ and $\mu_{s}$ is singular to $m$. Define $E \mu=\mu_{s}$. It must be verified that $E$ is the required $L$-projection.

It is clear that $\|\mu\|=\|E \mu\|+\|\mu-E \mu\|$ so we only have to show $E\left(H^{\infty}\right)^{\perp}=$ $\left(H^{\infty}+C\right)^{\perp}$. The Abstract F. and M. Riesz Theorem, as given in $[4$, p. 44, Corollary 7.9], takes the following form when applied to $H^{\infty}$.

TheOREM 3.2. Let $H_{0}^{\infty}=\left\{f \in H^{\infty}: \int f d m=0\right\}$ and $\left(H_{0}^{\infty}\right)^{\perp}=\left\{\mu \in C(M)^{*}\right.$ : $\int f d \mu=0$ for all $\left.f \in H_{0}^{\infty}\right\}$. Let $\mu \in\left(H_{0}^{\infty}\right)^{\perp}$ be written $\mu=\mu_{a}+\mu_{s}$ where $\mu_{a} \ll m$ and $\mu_{s} \perp m$; then $\mu_{a} \in\left(H_{0}^{\infty}\right)^{\perp}$ and $\mu_{s} \in\left(H^{\infty}\right)^{\perp}$.

Now, if $\mu \in\left(H^{\infty}\right)^{\perp}$ then $\bar{z} \mu \in\left(H_{0}^{\infty}\right)^{\perp}$, where $\bar{z}$ is the (Gelfand transform of) the function on the unit circle which takes $e^{i \theta}$ to $e^{-i \theta}$. If $\mu$ is singular with respect to $m$ so is $\bar{z} \mu$ and Theorem 3.2 implies $\bar{z} \mu \in\left(H^{\infty}\right)^{\perp}$. Repetition of this argument gives $\bar{z}^{n} \mu \in\left(H^{\infty}\right)^{\perp}$, that is

$$
\int \bar{z}^{n} f d \mu=0 \text { for all } f \in H^{\infty}, n>0 .
$$

Since $\cup_{n=0}^{\infty} \bar{z}^{n} H^{\infty}$ is dense in $H^{\infty}+C$ we see that $\mu \in\left(H^{\infty}+C\right)^{\perp}$ provided $\mu \in\left(H^{\infty}\right)^{\perp}$ and $\mu \perp m$. If $\mu$ is an arbitrary element of $\left(H^{\infty}\right)^{\perp}$ then Theorem 3.2 implies $E \mu \in\left(H^{\infty}\right)^{\perp}$. Since $E \mu \perp m$ we get $E \mu \in\left(H^{\infty}+C\right)^{\perp}$.

To show that $E$ is onto we show that every $\mu \in\left(H^{\infty}+C\right)^{\perp}$ is singular with respect to $m$. Suppose $\mu=\mu_{a}+\mu_{s}$ as before. Then $\mu_{s} \in\left(H^{\infty}+C\right)^{\perp}$ again, and so $\mu_{a} \in\left(H^{\infty}+C\right)^{\perp}$. Writing $\mu_{a}=\hat{h} d m$ with $h \in L^{1}(d \theta)$ this implies $\int g h d \theta=0$ for all continuous $g$. Thus $h=0$ and $\mu_{a}=0$, i.e., $\mu=E \mu$ and $E$ is onto.

4. Remarks. It would be interesting to know if the analogues of Theorem 1.1 and Theorem 2.4 are true with $H^{\infty}+C$ replaced by other subalgebras of $L^{\infty}$ containing $H^{\infty}$.

The best approximation guaranteed by Theorem 1.1 is not unique (in fact is never unique) if $f \notin H^{\infty}+C$. This was obtained in [3] and can also be deduced from Theorem 2.4 using results on $M$-ideals by Holmes, Scranton, and Ward [7].

\section{REFERENCES}

1. V. M. Adamjan, D. Z. Arov and M. G. Krein, Analytic properties of Schmidt pairs for a Hankel operator and the generalized Shur-Takagi problem, Mat. Sb. 86 (128) (1971), 34-75 = Math. USSR Sb. 15 (1971), 31-73.

2. E. Alfsen and E. Effros, Structure in real Banach spaces, Ann. of Math. 96(1972), 98-173.

3. S. Axler, I. D. Berg, N. Jewell and A. Shields, Approximation by compact operators and the space $H^{\infty}+C$, Ann. of Math. (2) 109 (1979), 601-612.

4. T. Gamelin, Uniform algebras, Prentice-Hall, Englewood Cliffs, N. J., 1969.

5. K. Hoffman, Analytic functions and logmodular Banach algebras, Acta Math. 108 (1962), 271-317.

6. __ Banach spaces of analytic functions, Prentice-Hall, Englewood Cliffs, N. J., 1962.

7. R. Holmes, B. Scranton and J. Ward, Approximation from the space of compact operators and other M-ideals, Duke Math. J. 42 (1975), 259-269.

8. Z. Nehari, On bounded linear forms, Ann. of Math. 65 (1957), 153-162.

9. D. Sarason, Generalized interpolation in $H^{\infty}$, Trans. Amer. Math. Soc. 127 (1967), 179-203.

Department of Mathematics, Michigan State University, East Lansing, Michigan 48824 